\title{
A Smart Home System based on Microcontroller using Android Application and MySQL Database
}

\author{
Uğur Bayrak, Ömer Gül \\ Istanbul Technical University, Department of Electrical Engineering, Maslak, Istanbul 34467, Turkey
}

\begin{abstract}
In recent years, people may disremember to turn off various devices or not be sure open or closed. Predefined scenarios for these people, home comfort and efficiency in the workplace will help a lot. Most people spend time with mobile phones throughout the day. Therefore, the control of smart devices by phones would be an appropriate solution. In this study, it is designed with microcontroller based intelligent building automation system hardware and software to provide energy efficient, security and comfort. The programming of the microcontroller, writing the android application program for the mobile phone and MySQL database software were realized. Microcontroller with GSM shield, it is possible to control the devices connected to the system by an android application automatically and manually by means of the internet connection by the MySQL database on the remote server. It is ensured that all devices are adjusted with a single touch according to predefined scenarios.
\end{abstract}

Key words: Database systems, design automation, microcontrollers, sensor systems, smart homes

\section{Introduction}

A Smart home application is an attractive area that provides benefits such as additional comfort, increased security and efficient energy use. The operation of a home automation system focused on the key tasks of turn on/off different devices remotely. Recent technological advances have allowed researchers to use Wi-Fi technology to connect different devices in a home automation system.

The smart home system includes many smart features to make life easier. When we remember that we have forgotten some devices such as oven, toaster and lamp, we can turn off to these devices by using our mobile phone or internet. If the system detects unauthorized transactions at home, it sends messages to our mobile phones. The only limitation for you is the budget and your dreams for automation. With the development of technology, things in daily life are becoming much easier for us. An internet-based home automation system focuses on controlling home electronic devices, whether someone is inside or outside the home.

Developed internet-based home automation system users can access the network via their websites to see the status of their homes and their families. Researchers have developed a home automation system based on SMS technology with the use of mobile phones [1]. In recent years, with the development of technology, home automation has become a part of our daily lives. We can control any devices from anywhere with internet-based systems. A microcontroller-based home automation system that uses an internet server has been used to monitor and control household devices [2]. A Bluetooth based home automation system that uses the mobile phone considering that people no longer have their phones away at all [3]. In another home automation system, it is

*Corresponding author: Address: Faculty of Engineering, Department of Civil Engineering Sakarya University, 54187, Sakarya TURKEY. E-mail address: caglar@ sakarya.edu.tr, Phone: +902642955752 
equipped with a voice activation system to serve persons with disabilities and elderly people who cannot self-care using Wi-Fi technology [4]. In another study, a home automation system based on Bluetooth wireless technology and mobile phone, which enables the monitoring and control of different networked devices, has been dealt with [5]. A smart home control and monitoring system has been implemented using the android application and a built-in micro web server to remotely access and control various devices [6]. In another study, an iCloud-based home automation system has been developed to precisely monitor many household appliances [7].

A home automation system was implemented using GSM, internet and speech recognition. In this system, the home gateway requires a personal computer and it is difficult to keep the system open at all times due to energy consumption to manage the automation system [8,9]. Embodied Conversational Agent based interfaces on android developed a prototype installed on a tablet for controlling household devices [10].

In another study, the system designed a home power management aimed at reducing energy consumption [11]. This system also uses the cloud as a home gateway with limitations as described previously. A mobile based home automation system with Java support is described in [12]. Authors proposed a home-based ZigBee-based home network configuration that controls all home appliances with a ZigBee-infrared combination [13]. Lee et al. [14] proposed a wireless network protocol that make available a two-way communication channel between a gateway and a controller, highlighting of a wireless sensor network in the control of home appliances. The microcontroller-based home security system in [15] is designed without GSM technology. A microcontroller-based home security system with GSM technology is defined. A Bluetooth application was also used to control the system [16]

In this study, a low cost and secure, mobile phone based flexible home automation system has been realized. By using the microcontroller with GSM shield, it is ensured that the devices in the house via an Android application can be controlled automatically and manually. All devices can be adjusted with a single touch according to predefined scenarios. It is designed with microcontroller based intelligent building automation system hardware and software the objectives of the work and provide an adequate background, avoiding a detailed literature survey or a summary of the results [1].

\section{Automation System Components}

\subsection{Sensors}

The automation system makes use of five types of sensors to sense the environmental condition in the home. These are described briefly below.

Temperature and Humidity Sensor is used to measure the temperature and humidity values of the environment. The measured values are written to the database via the internet. The user can monitor these values via the phone application. When the ambient temperature increases, it is possible to reduce the temperature by running a fan via the telephone application. At the same time, the fan can be operated automatically to ensure that the heat is kept at the desired values.

The motion detection sensor is used to send an emergency message to the user when a motion is detected in the environment when no one is in the house and to allow the lamps to flash when night mode da is active. 

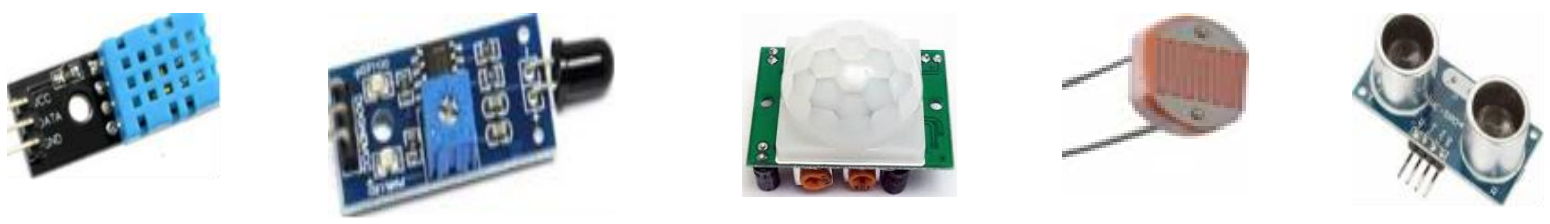

a) Temperature and Humidity Sensor, b) Flame Sensor c) Motion Sensor, d) LDR, e) Ultrasonic Distance Sensor

Figure 1. Sensors,

The motion detection sensor is used to send an emergency message to the user when a motion is detected in the environment when no one is in the house and to allow the lamps to flash when night mode da is active. The flame sensor allows the user to send an emergency signal when the flame is detected in the environment.

Two ultrasonic distance sensors were used to ensure asset control. When there is not enough light in the environment, the lamps work when an asset is detected. When a signal is applied to the Trig pin of the sensor, an ultrasonic sound wave is emitted at a frequency of $40 \mathrm{kHz}$. When the sound waves hit any object and returns to the sensor, the Echo pin outputs an output signal. In this way, the number of people entering and leaving the room is determined and lighting control is provided. In this project, the LDR was used to adjust the light intensity of the lamps depending on daylight. An LDR was placed next to each lamp, allowing the lamps to be dimmed in order to save energy in the event of excessive daylight. It gives 10-bit analog output value between 0-1023 according to light falling on LDR. While the output value decreases in the dark environment, the output value increases in bright environments. These output values are converted to 0-255 and the brightness of the lamps is adjusted. The value 0 corresponds to $0 \mathrm{~V}$ and the value 255 corresponds to $5 \mathrm{~V}$.

\subsection{Servomotor}

Servo motor is used for shutter control. This motor can rotate between 0 and 180 degrees with a precision of 1 degree. The shutter control is automatically switched on and off both manually and depending on daylight.

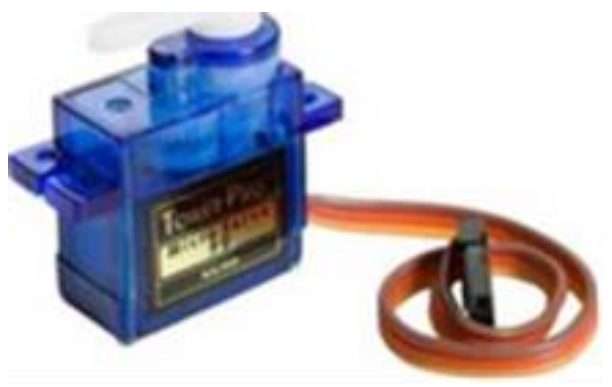

Figure 2. Servo-motor

\subsection{Microcontroller}

A microcontroller has been selected as an open source development platform based on easy-to-use hardware and software. There are 54 digital input / output pins and 16 analog input pins. 14 of the 
digital pins can be used as PWM outputs. It can communicate with the computer via a USB cable.

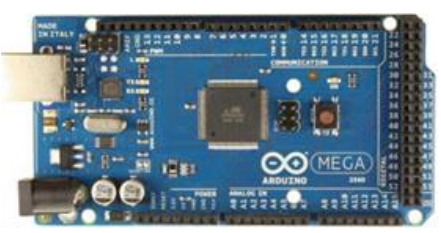

Figure 3. Microcontroller

\subsection{GSM Shield}

It enables the microcontroller to connect to the internet via the GPRS wireless network, send and receive SMS, and make calls. In this project, it is used to transfer the information of the lamps, fan and louver on-off status and brightness levels, temperature and humidity information, motion detection and information received from flame sensors to the database via internet. It will also send an emergency message to the designated phone number when one of the motion detection and flame sensors is triggered.

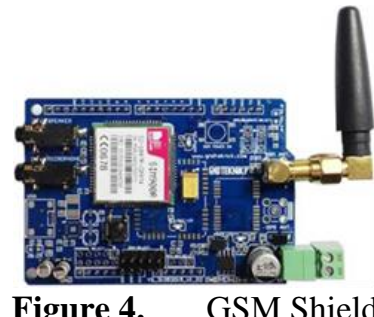

\section{Methods of Communication in Smart Home}

The language used to provide data transfer between the two devices is called the protocol. Protocols regulate the communication rules in a network. It is possible for the devices to communicate with each other in the same way if the protocols are used in the same way or in harmony with each other. In cases where a new device is added to a system that has been installed or if the system needs to be renewed, the user must be dependent on the brand installed in the system or prefer a different brand in order to meet the requirements. As the installed system cannot comply with the communication standard, it is necessary to either change the system installed depending on the brand that it would prefer or install a separate system for the devices to be added.

The building automation system consists of lighting, safety, energy monitoring, HVAC and fire systems. Thanks to open systems, a structure is created that allows these systems to communicate with each other. The major, open protocols are BACnet, LonWorks, Modbus, EIB, KNX, OPC, DALI. Wireless communication is the transmission of information between two or more points without any wired conductors. For many years' radio frequency has been widely used in wireless data transfer.

Infrared (IR) systems use high frequencies just below the visible light in the electromagnetic spectrum to transfer data. Infrared rays, such as light, cannot pass through opaque objects. The elements working with the infrared system must see each other. In addition, the communication distance is also very short as it is approximately $10 \mathrm{~m}$. 
Bluetooth is a wireless communication method developed as an alternative to wired communication, providing data transfer at close distances and providing high speed. The most noticeable difference between Bluetooth and other communication systems is that multiple devices can communicate with each other at the same time. With the widespread use of smart phones in recent years, the issue of control via Android devices has gained importance. In order to achieve this control, the software part contains the application development part for android. The java programming language is often used for application development.

$\mathrm{Wi}-\mathrm{Fi}$ is a protocol that offers wireless internet access over radio frequency. Thanks to its highspeed infrastructure, it is suitable for internet access where speed is important, but it is not suitable for sensor networks. For sensor networks, not only speed, but also cost, distance, ease of use, energy consumption are important. The frequencies at which it operates do not require a license, do not require a cable for the network, so that it is very difficult to pull cable. In networks with multiple wireless access points, wireless communication can be provided from one point to another without interruption.

The aim of the ZigBee protocol is to provide wireless communication in a wide coverage area with little energy consumption. In ZigBee technology, it is an advantage that the network topology is flexible according to most communication methods. Networking with mesh topology provides flexibility. Healthy and controlled communication can be achieved in a wide coverage area. ZigBee's data transfer speed, low power consumption, coverage, safety standards and topology flexibility make it suitable for wireless sensor networks and intelligent buildings. ZigBee's data transfer rate is more limited than Bluetooth

\section{Home Automation Software Design}

The programming of the microcontroller, writing the android application program and MySQL database software were realized. Microcontroller with GSM shield, it is possible to control the devices connected to the system via an android application automatically and manually via the internet connection by the MySQL database on the remote server.

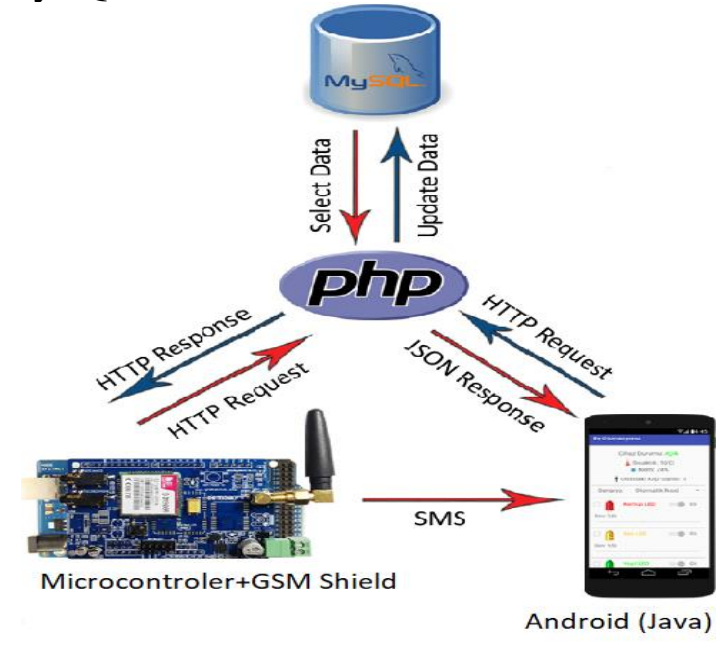

Figure 5. Figure Smart home automation system block diagram 


\subsection{Microcontroller programming}

To program the microcontroller, libraries and variables are defined first. Then, communication speeds and pin used in the setup function are defined. When the microcontroller starts up, it reads the codes in the setup function first. The codes in this function are read only once. In this area, it is stated which lamps, ultrasonic sensors, motion sensors, flame sensors, servo motors, temperature and humidity sensors use and which operating mode they use.

After the setup function is read, the loop function is read. The loop function is an infinite loop. The main program codes are written in this function. Lamps, fans and servo motors are controlled according to data in this field. At the same time, the data from the sensors are printed on the database.

The control of the lamps is provided both manually and automatically according to the data from the database. Automatic control is done with ultrasonic sensors and LDRs. Two ultrasonic sensors were used for asset control. First, the number of people entering and leaving the room is determined depending on which sensor is triggered. For each lamp, an analog output is obtained according to the luminous level of the environment with the LDRs that are placed separately. If this analog output value is 600 or smaller and the number of people in the room is greater than zero, the lamps will be activated. Otherwise, the lamps will be disabled.

The fan control can be controlled manually or automatically according to the data from the database. In the automatic control, the temperature measured by the DHT11 temperature and humidity sensor is determined by comparing the temperature read from the database to the maximum temperature determined by the telephone application. If the maximum temperature value is smaller than the temperature measured by the DHT11 sensor, the fan runs. The fan continues to run until the measured temperature falls below the maximum temperature value. Otherwise, the fan stops running.

The servo motor control used for the louver is also provided manually and automatically. Automatic control is performed with LDR. When the environment is bright, the blinds are opened and the shutter is closed when it is dark. In this way, daylight can be used.

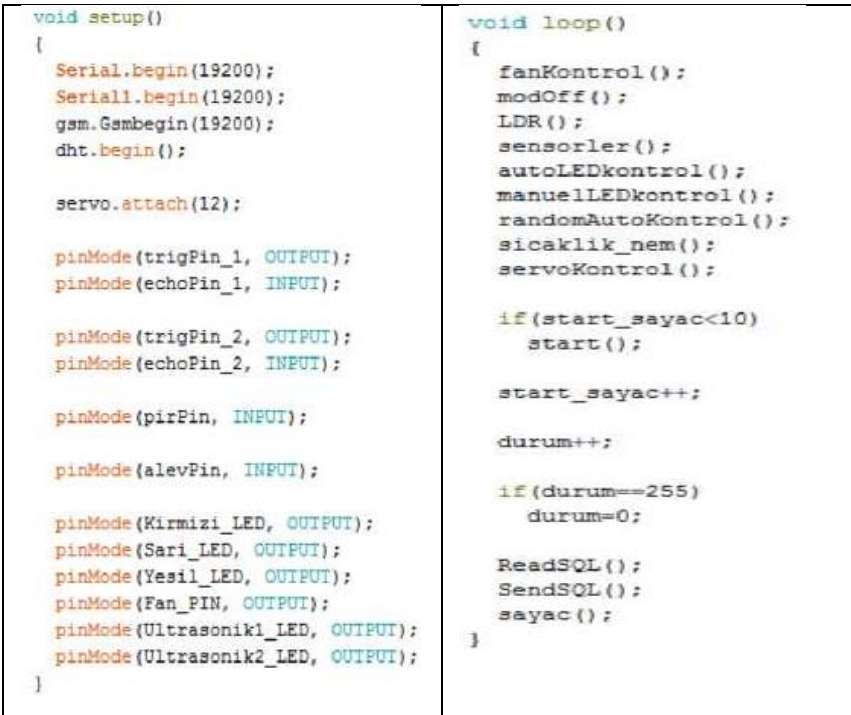

Figure 6. Microcontroller setup and loop function 
When the motion and flame sensor is triggered, an emergency message is sent to the specified phone number. The flame sensor is always active. The motion sensor is activated when the seç "Home leave "or" Holiday "scenarios are selected from the telephone application.

\subsection{Android application programming}

A phone application has been written for the android operating system in order to control the system via internet connection. The application is written in the Android Studio program, Android application development platform using Java programming language.

Predefined scenarios allow the entire system to be controlled with a single tap. There are five different scenarios, including automatic mode, special mode, night mode, home leave and holiday mode.

Auto Mode: In this scenario, the lamps are switched on and off automatically according to the number of people in the room and the brightness level of the environment. If there is at least one person in the room and the analog output value obtained from the LDR is 600 or less, the lamps are turned on. Otherwise the lamps are switched off. The fan is controlled according to the maximum temperature value determined by the application. If the specified maximum temperature value is equal to or lower than the temperature measured by the DHT11 sensor, the fan operates to reduce the ambient temperature to the desired value. If the set maximum temperature value is greater than the temperature measured by the sensor, the fan is deactivated. The shutter is automatically opened and closed according to the brightness level of the environment. When the environment is bright, the blinds are opened in order to take advantage of the daylight and it is turned off automatically when it is dark.

Special Mode: In this scenario, the whole system can be controlled automatically and the desired devices can be controlled manually. If one of the lamps is to be controlled regardless of the number of people in the room and the brightness level of the room, manual control is provided by selecting the corresponding box. In the same way, manual control can be provided by selecting the corresponding boxes in the fan and blinds.

Night Mode: In this scenario, the dimmer level of the lambs is reduced to $30 \%$ to prevent glare and the shutter is closed if it is open. When motion is detected, the lamps are turned on, then turned off automatically. 


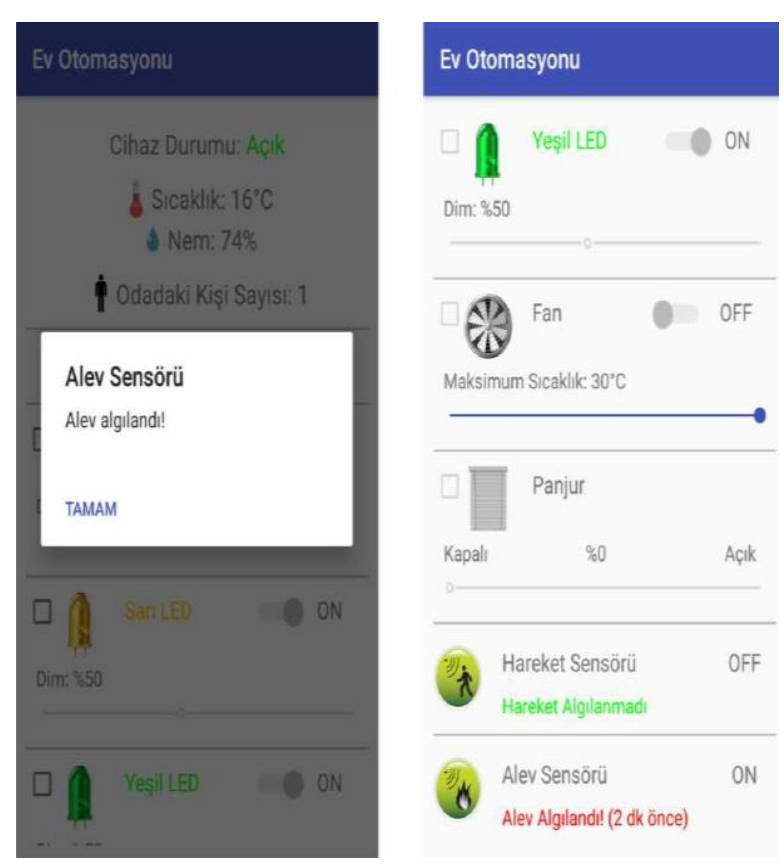

Figure 7. Home Automation Phone Application

Leave House: In this scenario all lamps, fan and shutter are closed. The motion detection sensor is activated. None of the devices will work until the user changes the scenario.

Holiday Mode: This scenario is suitable for use when the user is away from home for a long time. In this scenario, the entity is simulated to ensure the security of the house. The lamps and louvers are opened automatically at irregular intervals to try to create the impression that there is someone living in the house. If a motion or flame is detected, an emergency message is sent to the specified number. It can also be seen whether a movement or flame has been detected through the application.

\section{MySQL Database}

MySQL database was used to provide remote control of lamps, fan and louver, information on ambient temperature and humidity, whether any movement or flame was detected. The database is located on a remote server. PhpMyAdmin was used to create and manage MySQL database with internet connection.

The PHP programming language is used to write and read data into the database. Database and PHP codes are on the same server. When you want to control any Lamps, fan or louver with the android application, the data is sent to the PHP file running on the remote server with internet connection. The code in the PHP file works by reading the data from the phone application and sent to the MySQL database. 


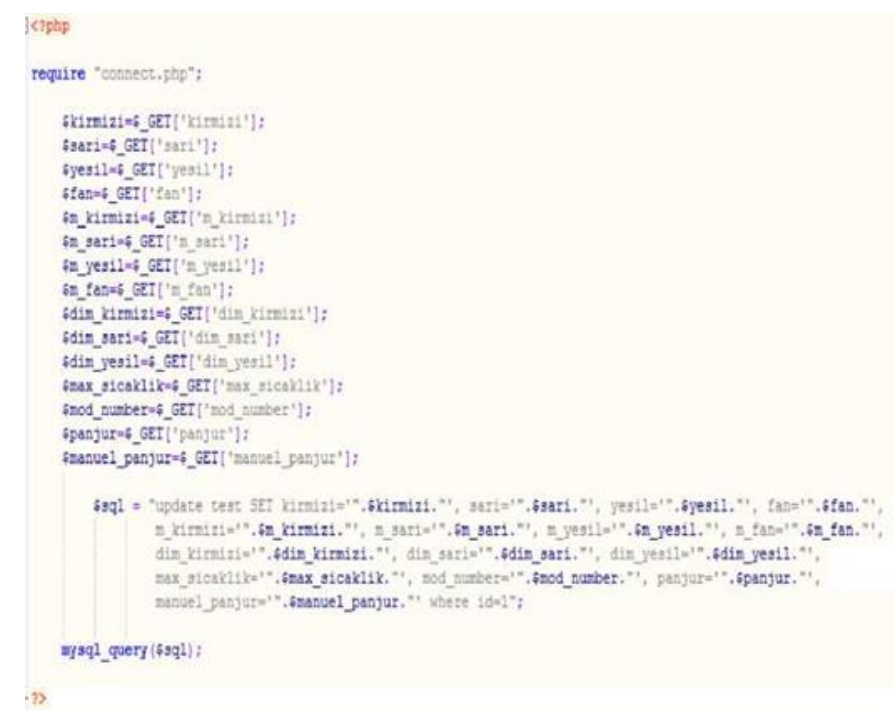

Figure 8. $\quad$ PHP codes used to print data into a MySQL database

The PHP codes are used to read the data which is sent to the database by the android application by the microcontroller. The microcontroller connects to the Internet at regular intervals to run the file containing these PHP codes. PHP codes print data on the screen. These data are read by the microcontroller and the relevant devices are checked.

\section{Conclusion}

Home automation has become one of the rapidly growing industries by changing lifestyles as well as meeting people's needs.

In this study, it is provided to control the devices connected to the system via an android application using microcontroller with GSM shield. Energy saving was achieved by turn on/off lightings by controlling the asset with ultrasonic sensors and adjusting the brightness by daylight with LDR. In addition, automatic louver control is provided with LDR depending on daylight to benefit from daylight. Temperature and humidity values of the environment can be monitored via the telephone application and automatic fan control can be performed to keep the ambient temperature at the desired value.

Using the motion and flame sensor, an emergency message is sent to the telephone number specified in cases such as a fire or theft when no one is at home. Besides, all devices can be adjusted with a single touch according to pre-prepared scenarios (automatic, special, night, home leave, holiday).

Energy saving was achieved by preventing unnecessary use of lighting with micro controller based building automation system, security of life and property in cases of theft and fire and user comfort is ensured by automatic or remote control of the system.

For the smart home automation application, the Microcontroller programming codes, Android Application Codes and MySQL PHP Codes are made from 1735 lines and 48544 characters (no spaces) coding. 


\section{References}

[1] H. ElKamchouchi and A. ElShafee, "Design and Prototype Implementation of SMS Based Home Automation System," 2012 IEEE International Conference on Electronics Design, Systems and Applications (ICEDSA).

[2] N. David, A. Chima, A. Ugochukwu and E. Obinna, "Design of a home automation system using arduino," International Journal of Scientific \& Engineering Research, vol. 6, Issue 6, pp. 795-801, June- 2015.

[3] R.Piyare and M.Tazil, "Bluetooth Based Home Automation System using Cell-phone," 2011 IEEE 15th International Symposium on Consumer Electronics.

[4] Shiu Kumar, "Ubiquitous Smart Home System Using Android Application," International Journal of Computer Networks \& Communications (IJCNC), vol. 6, no. 1, pp. 33-43, January 2014.

[5] M. O. A. Bader, I. M. A. Iman, H. M. A. Mahdi, H. A. A. Sami and S. S. Mohamed, "Design and Implementation of a Reliable Wireless Real-Time Home Automation System Based on Arduino Uno Single-Board Microcontroller,'International journal of control, Automation and systems, vol. 3, no. 3, pp. 11-15, 2014.

[6] R. Piyare1 and S. R. Lee1, "Smart Home-Control and Monitoring System Using Smart Phone", Independent Computer Consultants Association 2013, ASTL vol. 24, pp 83 - 86, 2013 (C) SERSC 2013.

[7] M. G. Golzar and H. Tajozzakerin, "A New Intelligent Remote-Control System for Home Automation and Reduce Energy Consumption," 2010 Fourth Asia International Conference on Mathematical/Analytical Modelling and Computer Simulation.

[8] El-Medany, W.M. and El-Sabry, M.R. (2008) GSM-Based Remote Sensing and Control System using FPGA. Proceedings of International Conference on Computer and Communication Engineering, Kuala Lumpur, 13-15 May 2008, 1093-1097.

[9] Yuksekkaya, B., Kayalar, A.A., Tosun, M.B., Ozcan, M.K. and Alkar, A.Z. (2006) A GSM, Internet and Speech Controlled Wireless Interactive Home Automation System. IEEE Transactions on Consumer Electronics, 52, 837-843.

[10] M. Santos-Perez, E. Gonzalez-Parada and J. M. C. Garcia, "ECA based Control Interface on Android for Home Automation System," 2013 IEEE International Conference on Consumer Electronics (ICCE).

[11] Golzar, M.G. and Tajozzakerin, H.R. (2010) A New Intelligent Remote-Control System for Home Automation and Reduce Energy Consumption. 4th Asia International Conference on Mathematical/Analytical Modelling and Computer Simulation, Kota Kinabalu, 26-28 May 2010, 174-180.

[12] Van Der Werff, M., Gui, X. and Xu, W.L. (2005) A Mobile-Based Home Automation System. Proceedings of the 2nd International Conference on Mobile Technology, Applications and Systems, Guangzhou, 15-17 November 2005, 1-5.

[13] Hwang, I.-K., Lee, D.-S. and Baek, J.-W. (2009) Home Network Configuring Scheme for All Electric Appliances using ZigBee-Based Integrated Remote Controller. IEEE Transactions on Consumer Electronics, 55, 13001307.

[14] Lee, H.-B., Park, J.-L., Park, S.-W., Chung, T.-Y. and Moon, J.-H. (2010) Interactive Remote Control of Legacy Home Appliances through a Virtually Wired Sensor Network. IEEE Transactions on Consumer Electronics, 56, 2241 2248.

[15] Islam, M.S. (2014) Home Security System Based on PIC18F452 Microcontroller. Proceedings of 2014 IEEE International Conference on Electro/Information Technology, Milwaukee, 5-7 June 2014, 202-205.

[16] Hasan, R. , Khan, M. , Ashek, A. and Rumpa, I. (2015) Microcontroller Based Home Security System with GSM Technology. Open Journal of Safety Science and Technology, 5, 55-62. 